\title{
DIFERENCIAS DE GÉNERO EN LA EJECUCION DE HABILIDADES MOTORAS BASICAS EN PRE ESCOLARES CHILENOS
}

\section{DIFFERENCES FROM KIND IN THE EXECUTION OF SKILLS MOTOR BASIC IN PRE - SCHOOL CHILEAN}

\author{
Dr. Gamboa Jiménez, Rodrigo
}

Pontificia Universidad Católica de Valparaíso, Escuela de Educación Física

\begin{abstract}
GAMBOA J.R. Diferencias de género en la ejecución de habilidades motoras básicas en pre escolares chilenos Mot. Hum., 10(2): 87-94, 2010.

\section{RESUMEN}

El presente artículo analiza si existen diferencias estadísticamente significativas, según género, en la ejecución de las habilidades motoras básicas en una muestra conformada por 276 estudiantes de una poblacióm total de 1.333 niños y niñas de 4 y 5 años de edad que asisten a jardines de infante pertenecientes a INTEGRA, JUNJI y Ministerio de Educación de la ciudad de Viña del Mar. Se evalúan habilidades de locomoción, manipulación y equilibrio o estabilidad por medio de una pauta de observación confeccionada y validada en pre escolares de la Región del Maule, Chile, Vargas, C. (1). En el análisis de los datos, a través de un análisis ANOVA con un nivel de significacncia alfa =0,005, en el cual se demuestra que no se observan diferencias estadísticamente significativas, en la variable género, en la muestra total del estudio, sólo algunos casos puntuales al interior del estamento educacional JUNJI.
\end{abstract}

PALABRAS CLAVE: Pre escolares, patrones fundamentales de movimiento, evaluación, diferencias significativas, género.

\section{INTRODUCCION}

En Chile, las investigaciones sobre habilidades motoras básicas en los últimos años son insuficientes, y por tanto, no existe una gama amplia de publicaciones a las cuales remitirse para recopilar información sobre la temática en niños y niñas chilenas o de alguna región del país. Destaca fundamentalmente la investigación realizada por Vargas, C. (1). En ella se hace un estudio descriptivo de la presencia y ausencia de estas habilidades a nivel parvulario de la Región del Maule (INTEGRA, JUNJI y Ministerio de Educación).

Es importante conocer y comprender como evoluciona la motricidad, especialmente en la infancia, y de esta manera poder responder a interrogantes relevantes para la Educación Física escolar, tales como: ¿cuáles son las características motrices de niños y niñas?, ¿existen diferencias en estas características según gënero?, ¿cuáles son las etapas por las que pasan para poder lograr movimientos de calidad y lograr por ende autonomía?. Esto sin duda, permite responder a preguntas esenciales dentro del quehacer de la educación física que tienen relación con: ¿qué objetivos trabajar en la infancia?; ¿cuáles son las tareas que ayudarán a lograr estos objetivos?; y ¿cómo evaluar la motricidad en los niños y niñas?

En el contexto de esta evolución motriz, ambiente, maduración y herencia, son tres factores que inciden de manera positiva o negativa en los procesos de desarrollo de la motricidad de las personas, donde el resultado, es producto de la interacción conjunta de los tres factores mencionados, donde se le otorga vital importancia a los efectos del ambiente y procesos madurativos, $(2,3,4,5)$.

La presente investigación ha centrado su atención en los efectos del ambiente en el desarrollo de la motricidad en niños y niñas, especificamente en las diferencias de género.

El ambiente, hace referencia fundamentalmente al medio o entorno sociocultural en que se encuentran inmersas las personas. Siendo considerado como un factor de vital importancia y que es considerado por varios autores como el que más incide en el desarrollo motor de las personas, $(3,6,7,8,9)$ 
GAMBOA J.R. Diferencias de género en la ejecución de habilidades motoras básicas en pre escolares chilenos Mot. Hum., 10(2): 87-94, 2010.

El desarrollo motor en los primeros años de vida se da de manera muy rápida, y es de consenso de todos quienes se relacionan con el área, que la infancia es su etapa crítica de desarrollo, y el medio donde se desenvuelve el niño o la niña, cobra un rol fundamental en este proceso, Mc Clenaghan y Gallahue (4). En relación a este ambiente, se presenta un elemento sensible y de suma relevancia, y que se relaciona con las diferencias que se realizan entre géneros, repercutiendo en el qué y cómo aprenden los niños y las niñas, $(10,11,12)$.

Estas diferencias entre géneros en el desarrollo y aprendizaje motor, son producidas fundamentalmente por el trato diferenciado y las expectativas sociales que la sociedad plantea para los niños y para las niñas, $(13,14)$. El propio Thomas, en una revisión que realizó de 64 estudios sobre 20 habilidades motoras, concluye que hay diferencias de género, en algunas tareas como el lanzar, el bolear, agilidad y tiempo de reacción a favor de los niños, y en otras relacionadas con coordinación óculo manual y flexibilidad, a favor de las niñas.

García (10), extrapola estas diferencias entre género al plano de la educación formal, al plantear que lo que aprenden y como aprenden en el jardín de infantes los niños y las niñas, está condicionado socialmente por el género.

En la relación entre la cultura y la enseñanza, estas diferencias seguirán existiendo en la medida que las tareas y oportunidades de práctica motriz estén condicionadas por las fuertes expectativas que posee la sociedad en relación a la competencia motriz que deben adquirir tanto niñas como niños. Y en la educación, el género es un tema de preocupación y es necesario atender, debido a que trasciende desde la sociedad, y desde siempre, socialmente las mujeres han sido ubicadas en un segundo plano.

Se deben realizar esfuerzos para realizar cambios que no sólo sean de forma, no se trata solamente que niños y niñas se eduquen juntos como es el caso de la educación mixta, si no que se debe buscar soluciones de fondo a través de la igualdad de oportunidades. THOMAS, THOMAS y WILLIAMS, 2008 (15), plantean que no hay razones, en relación al desarrollo, como para realizar diferencias entre lo que se les enseña, como se les enseña y lo que deben aprenden los niños y las niñas.

La clase de educación física debe ayudar a no establecer estas diferencias entre lo que deben hacer y aprender, en este caso en relación a la motricidad de los niños y las niñas. La educación debe dar igualdad de oportunidades, y no el modelo curricular de los niños se debe adaptar a las niñas para que ellas participen. Este es un desafió grande al cual debe responder la educación, los centros educativos, los docentes, y especialmente, los docentes de educación física, se debe favorecer una participación activa de todos los niños y las niñas.

\section{MATERIAL Y METODO.}

Se ha decidido por una muestra de tipo probabilística estratificada con asignación de Neyman con el propósito de que la muestra sea proporcional a como se presenta en la población. Se han establecido 12 estratos en la muestra según sexo edad y estamento educacional, y esta conformada por 276 niños y niñas entre 4 y 5 años de edad de la ciudad de Viña del Mar pertenecientes a los jardines infantiles INTEGRA, JUNJI y Ministerio de Educación. La muestra representa el 20,70\% de la Población que esta compuesta por 1.333 niños y niñas.

La muestra se desglosa de la siguiente manera: INTEGRA representa el $10,5 \%$ de la muestra estudiada y esta conformada por cuatro estratos: 16 niños y 13 niñas de 4 años de de edad, y 0 niños y 0 niñas de 5 años de edad. JUNJI representa el 43,1\% de la muestra estudiada compuesta por cuatro estratos: 52 niños y 47 niñas de 4 años de edad, y 11 niños y 9 niñas de 5 años de edad. y Ministerio de Educación, que representa el $46,4 \%$ de la muestra estudiada y se subdivide en el mismo número de estratos. 32 niños y 22 niñas de 4 años de edad, y 35 niños y 39 niñas de 5 años de edad.

Para comprobar si existen diferencias estadísticamente significativas en las variables género en la realización de las habilidades motoras básicas, se ha utilizado la pauta de observación elaborada y validada por Cesar Vargas Vitoria, en su tesis Doctoral, Vargas (2004). Posee 8 dimensiones, y cada una de ellas posee sus Variables a observar, que en total suman 43: marcha (9 variables), cuadrupedia (6 variables), vertical (subir y bajar 
GAMBOA J.R. Diferencias de género en la ejecución de habilidades motoras básicas en pre escolares chilenos Mot. Hum., 10(2): 87-94, 2010.

escaleras, 4 variables), carrera (4 variables),, salto (9 variables), lanzamiento (5 variables), golpear (4 variables) y equilibrio (2 variables). Que permite evaluar el grado de presencia o ausencia de estas habilidades agrupadas en las dimensiones anteriormente mencionadas.

El análisis estadístico de los datos se realizó a través de la pruebas estadísticas paramétricas Análisis
ANOVA. Para tal efecto, se utilizó el paquete estadístico SPSS. Se entenderá que el nivel de significancia empleado es alfa $=0,05$ y se comparan los valores obtenidos en cada una de las dimensiones referentes a las habilidades motoras básicas para comprobar con respecto a 0,05 , si la diferencia es o no estadísticamente significativa al $5 \%$ en la variable género.

\section{RESULTADOS}

ANOVA $^{\mathrm{a}}$

\begin{tabular}{|c|c|c|c|c|c|c|}
\hline & & $\begin{array}{c}\text { Suma de } \\
\text { cuadrados }\end{array}$ & $\overline{\text { gl }}$ & $\begin{array}{c}\text { Media } \\
\text { cuadrática }\end{array}$ & $\bar{f}$ & Sig \\
\hline \multirow[t]{3}{*}{ Equilibrio } & Inter-grupo & 0,656 & 1 & 0,656 & 1,453 & 0,229 \\
\hline & Intra-grupo & 123,703 & 274 & 0,451 & & \\
\hline & Total & 124,353 & 275 & & & \\
\hline \multirow[t]{3}{*}{ Cuadrupedia } & Inter-grupo & 0,002 & 1 & 0,003 & 0,001 & 0,970 \\
\hline & Intra-grupo & 345,477 & 274 & 1,261 & & \\
\hline & Total & 345,478 & 275 & & & \\
\hline \multirow[t]{3}{*}{ Marcha } & Inter-grupo & 0,233 & 1 & 2,330 & 0,197 & 0,658 \\
\hline & Intra-grupo & 323,767 & 274 & 1,182 & & \\
\hline & Total & 324,000 & 275 & & & \\
\hline \multirow[t]{3}{*}{ Vertical } & Inter-grupo & 1,707 & 1 & 1,707 & 1,253 & 0,263 \\
\hline & Intra-grupo & 371,377 & 274 & 1,355 & & \\
\hline & Total & 373,083 & 275 & & & \\
\hline \multirow[t]{3}{*}{ salto } & Inter-grupo & 6,650 & 1 & 6,650 & 1,141 & 0,286 \\
\hline & Intra-grupo & 1537,042 & 274 & 5,829 & & \\
\hline & Total & 1603,692 & 275 & & & \\
\hline \multirow[t]{3}{*}{ Lanzamiento } & Inter-grupo & 3,396 & 1 & 3,396 & 3,155 & 0,077 \\
\hline & Intra-grupo & 294,962 & 274 & 1,077 & & \\
\hline & Total & 298,359 & 275 & & & \\
\hline \multirow[t]{3}{*}{ Carrera } & Inter-grupo & 0,258 & 1 & 0,258 & 0,360 & 0,549 \\
\hline & Intra-grupo & 196,130 & 274 & 0,716 & & \\
\hline & Total & 196,388 & 275 & & & \\
\hline \multirow[t]{3}{*}{ Golpear } & Inter-grupo & 1,577 & 1 & 1,577 & 1,820 & 0,178 \\
\hline & Intra-grupo & 237,391 & 274 & 0,866 & & \\
\hline & Total & 238,967 & 275 & & & \\
\hline
\end{tabular}

a NIVEL = Muestra

TABLA I: análisis ANOVA, diferencias significativas entre niñas y niños del total de la muestra

Se puede observar en la tabla I, que no existe diferencias significativas según género, en las dimensiones objeto de estudio. 
GAMBOA J.R. Diferencias de género en la ejecución de habilidades motoras básicas en pre escolares chilenos Mot. Hum., 10(2): 87-94, 2010.

ANOVA INTEGRA

\begin{tabular}{|c|c|c|c|c|c|c|}
\hline & & Suma de cuadrados & gl & Media cuadrática & $\mathbf{f}$ & Sig \\
\hline \multirow{3}{*}{ Equilibrio } & Inter-grupo & 0,121 & 1 & 0,121 & 0,305 & 0,585 \\
\hline & Intra-grupo & 10,707 & 27 & 0,397 & & \\
\hline & Total & 10,828 & 28 & & & \\
\hline \multirow{3}{*}{ Cuadrupedia } & Inter-grupo & 0,048 & 1 & 0,048 & 0,036 & 0,851 \\
\hline & Intra-grupo & 35,745 & 27 & 1,324 & & \\
\hline & Total & 35,793 & 28 & & & \\
\hline \multirow[t]{3}{*}{ Marcha } & Inter-grupo & 0,597 & 1 & 0,597 & 0,401 & 0,532 \\
\hline & Intra-grupo & 40,231 & 27 & 1,490 & & \\
\hline & Total & 40,828 & 28 & & & \\
\hline \multirow[t]{3}{*}{ Vertical } & Inter-grupo & 0,279 & 1 & 0,279 & 0,136 & 0,716 \\
\hline & Intra-grupo & 55,514 & 27 & 2,056 & & \\
\hline & Total & 55,793 & 28 & & & \\
\hline \multirow[t]{3}{*}{ salto } & Inter-grupo & 0,159 & 1 & 0,159 & 0,031 & 0,861 \\
\hline & Intra-grupo & 136,668 & 27 & 5,062 & & \\
\hline & Total & 136,828 & 28 & & & \\
\hline \multirow{3}{*}{ Lanzamiento } & Inter-grupo & 3,390 & 1 & 3,390 & 3,905 & 0,058 \\
\hline & Intra-grupo & 23,438 & 27 & 0,868 & & \\
\hline & Total & 26,828 & 28 & & & \\
\hline \multirow{3}{*}{ Carrera } & Inter-grupo & 0,520 & 1 & 0,520 & 0,861 & 0,362 \\
\hline & Intra-grupo & 16,308 & 27 & 0,604 & & \\
\hline & Total & 16,828 & 28 & & & \\
\hline \multirow{3}{*}{ Golpear } & Inter-grupo & 0,767 & 1 & 0,767 & 1,300 & 0,264 \\
\hline & Intra-grupo & 15,923 & 27 & 0,590 & & \\
\hline & Total & 16,690 & 28 & & & \\
\hline
\end{tabular}

TABLA II: análisis ANOVA, diferencias significativas entre niñas y niños pertenecientes a INTEGRA

Una vez analizada la tabla II, Podemos inferir que para el estamento educacional INTEGRA, no existe diferencias significativas en el en las dimensiones estudiadas para el presente estudio.

ANOVA JUNJI

\begin{tabular}{|c|c|c|c|c|c|c|}
\hline & & Suma de cuadrados & gl & Media cuadrática & $\mathbf{f}$ & Sig \\
\hline \multirow[t]{3}{*}{ Equilibrio } & Inter-grupo & ,747 & 1 & ,747 & 1,860 & 175 \\
\hline & Intra-grupo & 46,984 & 117 & ,402 & & \\
\hline & Total & 47,731 & 118 & & & \\
\hline \multirow[t]{3}{*}{ Cuadrupedia } & Inter-grupo & ,434 & 1 & ,434 & ,631 & ,428 \\
\hline & Intra-grupo & 80,490 & 117 & ,688 & & \\
\hline & Total & 80,924 & 118 & & & \\
\hline \multirow[t]{3}{*}{ Marcha } & Inter-grupo & 4,953 & 1 & 4,963 & 5,138 & 024 \\
\hline & Intra-grupo & 111,484 & 117 & ,953 & & \\
\hline & Total & 116,437 & 118 & & & \\
\hline \multirow[t]{3}{*}{ Vertical } & Inter-grupo & 7,471 & 1 & 7,471 & 7,660 & ,007 \\
\hline & Intra-grupo & 114,109 & 117 & 975 & & \\
\hline & Total & 121,580 & 118 & & & \\
\hline \multirow[t]{3}{*}{ salto } & Inter-grupo & 23,112 & 1 & 23,112 & 5,116 & ,026 \\
\hline & Intra-grupo & 528,585 & 117 & 4,518 & & \\
\hline & Total & 551,637 & 118 & & & \\
\hline \multirow[t]{3}{*}{ Lanzamiento } & Inter-grupo &, 622 & 1 & 622 & ,738 & ,352 \\
\hline & Intra-grupo & 98,538 & 117 &, 842 & & \\
\hline & Total & 99,160 & 118 & & & \\
\hline \multirow[t]{3}{*}{ Carrera } & Inter-grupo & 2.065 & 1 & 2,065 & 4,031 & 047 \\
\hline & Intra-grupo & 59,913 & 117 &, 512 & & \\
\hline & Total & 61,983 & 118 & & & \\
\hline \multirow[t]{3}{*}{ Golpear } & Inter-grupo &, 226 & 1 & ,226 & ,269 & ,605 \\
\hline & Intra-grupo & 98,413 & 117 & 841 & & \\
\hline & Total & 98,639 & 118 & & & \\
\hline
\end{tabular}

TABLA III: análisis ANOVA, diferencias significativas entre niñas y niños pertenecientes a JUNJI. 
GAMBOA J.R. Diferencias de género en la ejecución de habilidades motoras básicas en pre escolares chilenos Mot. Hum., 10(2): 87-94, 2010.

ANOVANINSTERIO DE EDUCACION

\begin{tabular}{|c|c|c|c|c|c|c|}
\hline & & Suma de cuadrados & gl & Media cuadrática & $\mathbf{f}$ & Sig \\
\hline \multirow[t]{3}{*}{ Equilibrio } & Inter-grupo & 0,273 & 1 & 0,279 & 0,550 & 0,460 \\
\hline & Intra-grupo & 63,940 & 126 & 0,507 & & \\
\hline & Total & 64,213 & 127 & & & \\
\hline \multirow[t]{3}{*}{ Cuadrupedia } & Inter-grupo & 0,329 & 1 & 0,329 & 0,191 & 0,663 \\
\hline & Intra-grupo & 217,725 & 126 & 1,728 & & \\
\hline & Total & 218,055 & 127 & & & \\
\hline \multirow[t]{3}{*}{ Marcha } & Inter-grupo & 3,166 & 1 & 3,166 & 2,677 & 0,104 \\
\hline & Intra-grupo & 149,013 & 126 & 1,183 & & \\
\hline & Total & 152,180 & 127 & & & \\
\hline \multirow{3}{*}{ Vertical } & Inter-grupo & 0,926 & 1 & 0,926 & 0,709 & 0,401 \\
\hline & Intra-grupo & 164,574 & 126 & 1,306 & & \\
\hline & Total & 165,500 & 127 & & & \\
\hline \multirow[t]{3}{*}{ salto } & Inter-grupo & 0,368 & 1 & 0,368 & 0,076 & 0,784 \\
\hline & Intra-grupo & 612,561 & 126 & 4,862 & & \\
\hline & Total & 612,930 & 127 & & & \\
\hline \multirow[t]{3}{*}{ Lanzamiento } & Inter-grupo & 1,233 & 1 & 1,233 & 1,041 & 0,319 \\
\hline & Intra-grupo & 149,260 & 126 & 1,185 & & \\
\hline & Total & 150,492 & 127 & & & \\
\hline \multirow[t]{3}{*}{ Carrera } & Inter-grupo & 0,064 & 1 & 0,064 & 0,074 & 0,786 \\
\hline & Intra-grupo & 108,866 & 126 & 0,864 & & \\
\hline & Total & 108,930 & 127 & & & \\
\hline \multirow[t]{3}{*}{ Golpear } & Inter-grupo & 3,432 & 1 & 3,432 & 3,812 & 0,053 \\
\hline & Intra-grupo & 113,443 & 126 & 0,900 & & \\
\hline & Total & 116,875 & 127 & & & \\
\hline
\end{tabular}

TABLA IV: análisis ANOVA, diferencias significativas entre niñas y niños pertenecientes a Ministerio de Educación

Si bien en la muestra total de estudio no existen diferencias estadísticamente significativas, cabe destacar que al interior del JUNJI, como se observa en la tabla III, si existe diferencias significativas en el género. Estas diferencia son a favor de los niños en las dimensiones marcha, vertical y salto, y a favor de las niñas en la dimensión carrera.

En el análisis de la tabla IV se observa, de la misma forma que los resultados emanados para INTEGRA y en la muestra total de estudio, en el estamento educacional Ministerio de Educación no existe diferencias significativas en el género en las dimensiones estudiadas.

\section{DISCUSIÓN}

A través del análisis ANOVA realizado para establecer si existen diferencias estadísticamente significativas entre género, en general esta panorámica no concuerda plenamente con lo planteado por diversos autores como García, Shala, y Lam y Shiller, $(10,16,17)$, al señalar en sus estudios que si existen tales diferencias, y que generalmente son a favor de los niños. Sin embargo, en el equilibrio o balance, estas diferencias son a favor de las niñas, lo cual tampoco se presenta en el presente estudio. Ejemplo de lo último mencionado, Lam y Shiller (17) y Shala (16), declaran en los resultados de sus investigaciones diferencias significativas a favor de las niñas en patrones de equilibrio, pero en aquellos donde se manipulan objetos, la diferencia es a favor de los varones.

Por su parte, Ruiz Pérez y Graupera (11), consideran que si bien, en edades tempranas es poca la diferencia entre genero, esta diferencia si es incrementada con la edad, debido principalmente al factor sociocultural.

Si bien en general, en los estudios hacen referencia a diferencias significativas principalmente a favor de los niños por sobre las niñas, es interesante mencionar lo que señalan Ruiz Pérez y Graupera (11), Torres (12); Thomas, Thomas y Williams (15), y Blázquez (18). Plantean que hay un fuerte arraigo sociocultural en los resultados que evidencian los diferentes estudios sobre esta temática. Niñas y 
GAMBOA J.R. Diferencias de género en la ejecución de habilidades motoras básicas en pre escolares chilenos Mot. Hum., 10(2): 87-94, 2010.

niños se les enseña, y ellos y ellas aprenden de manera diferente por causa de este aspecto social.

Frente a ete aspecto sociocultural, son interesante los resultados de la investigación de Nikolic y IlicStosôvic (19), donde se evidencia diferencias entre género en test que evalúan aspectos neuromaduracionales a favor de las niñas por sobre los niños, sin embargo, al parecer, los estudios demuestran que aprenden menos. Esto explica aun más que el factor ambiental en las diferencias entre damas y varones, está a favor de los niños.

Finalmente es importante relevar que en el presente estudio, si bien en la muestra total de estudio no se presentan diferencias estadísticamente significativas, al interior del estamento educacional JUNJI si existen, estas son a favor de los niños en las dimensiones marcha, vertical y salto, y a favor de las niñas en la carrera. Esta última diferencia a favor de las niñas concuerda con los resultados obtenidos por Vargas, C. (1).

\section{CONCLUSIONES}

De lo resultados y discusión, hay dos elementos importantes a considerar. Por un lado, diferentes autores ya citados plantean en sus estudios que existen diferencias en la ejecución de las habilidades motoras básicas generalmente a favor de los varones, lo cual, no se evidencia en el presente estudio, a excepción de los casos específicos ya mencionados. Y por otro, sería interesante seguir indagando en el tema aumentando el rango de edades de la muestra, debido a que se debe considerar la magnitud de edad de este estudio, 4 y 5 años de edad, es un rango reducido. De modo dede poder evaluar nuevamente a los niños y niñas que conforman la presente muestra de estudio, de modo de verificar si se mantiene la tendencia presentada, o como plantean Ruiz Pérez y Graupera (11), que esta diferencia entre género aumenta con la edad.

De los resultados, se hace evidente la importancia que poseen los procesos educativos, en el sentido de dar las mismas posibilidades de práctica motriz a niños y niñas, de modo que puedan desarrollar su motricidad. Estos procesos no se pueden dejar al azar y es impensable bajo todo punto de vista, como lo señala Gallahue y Ozmun (9), dejarlos en las manos sólo de los procesos madurativos,

\section{REFERENCIAS BIBLIOGRAFICAS}

1. Vargas, C. (2004). Tesis Doctoral. Pauta de observación de patrones motores en niños de 4 a 5 años: diseño, validación, observación de desarrollo motor y propuesta de estimulación. Universidad de Valladolid. Facultad de Educación y Trabajo Social. Departamento de Pedagogía. Valladolid.

2. Wickstrom, R. (1990). Patrones motores básicos. Madrid: Alianza Deportes.

3. Ruiz, L. (1994). Deporte y aprendizaje. Procesos de adquisición y desarrollo de habilidades. Madrid: Visor.

4. Mc Clenaghan, B. \& Gallahue, D. (1985). Movimientos fundamentales: su desarrollo $y$ rehabilitación. Buenos Aires: Medica Panamericana S.A.

5. Duarte M, Alves C, Buceen L. Desenvolvimento motor de pre - escolares no intervalo de 13 meses. Revista Brasileira de Cineantropometria \& Desempenho Humano 2005: 7 (2); 5 - 9.

6. Granda, J. \& Alemany, I. (2002). Manual de aprendizaje y desarrollo motor. Barcelona: Paidos.

7. Le Boulch, J. (1982). Hacia una ciencia del movimiento humano. introducción a la psicokinética. Buenos Aires: Paidos.

8. Gallahue, D. \& Ozmun, J. (1995) Motor development. children, infants, adolescent, adults. ( $2^{\mathrm{a}}$ ed). Indiana: Brown and Benchmark.

9. Gallahue, D. \& Ozmun, J. (2006). Understanding motor development. infants, children, adolescents, adults. ( $6^{\circ}$ ed). Indiana: Mc Graw Hill.

10. Garcia C. Gender differences in young children's interactions when laerning fundamental motor skills. Research Quarterly for Exercise and Sport 1994: 65 (3); 213-237. 
GAMBOA J.R. Diferencias de género en la ejecución de habilidades motoras básicas en pre escolares chilenos Mot. Hum., 10(2): 87-94, 2010.

11. Ruiz L, Graupera J. Competencia motriz y género entre los escolares españoles. En Revista Internacional de Medicina y Ciencias de la Actividad Física y del Deporte 2003: 3 (10); 101-111.

12. Torres D. Propuesta de programación didáctica coeducativo en educación física primaria: un caso de la sierra de madrid. En Retos: Nuevas Tendencias en E. F., Deporte Y Recreación 2005: 8; 43-49.

13.Canto, R. (2004). Comportamiento motor espontáneo en el patio de recreo escolar. Análisis de las Diferencias por Género en un Grupo de Escolares de 8 y 9 Años. Tesis Doctoral. Universidad Politécnica de Madrid. Facultad de la Actividad Física y el Deporte. Madrid.

14. Thomas J. Children's control, learning, and performance of motor skills. En Research Quarterly for Exercise and Sport 2000: 71 (1); $1-9$.

15. Thomas J, Thomas K, Williams K. Motor development and elementary physical education are partners. En Journal of Physical Education, Recreation \& Dance 2008: 79 (7); 40-43.

16. Shala M. Assessing gross motor skills of Kosovar preschool children. En Early Child Development and Care 2009: 179 (7); 969-976.

17. Lam H, Shiller W. A pilot study on the gross motor proficiency of hong kong preschoolers aged 5 to 6 years. En Early Child Development and Care 2001: 171 (1); 11-20.

18. Blázquez. D. (2006). Educación física. (2 $\left.{ }^{\mathrm{a}} \mathrm{ed}\right)$. Barcelona: Inde.

19. Nikolic S, Llic-Stosovic D. Detection and prevalence of motor skill disorders. En Research in Developmental Disabilities. 2009: 30 ; 12811287 
GAMBOA J.R. Diferencias de género en la ejecución de habilidades motoras básicas en pre escolares chilenos Mot. Hum., 10(2): 87-94, 2010.

\begin{abstract}
The present article analyzes if statistically significant differences exist, according to kind, in the execution of the skills basic motorboats in a sample shaped by 276 students of a total poblaciom of 1.333 children and 4 and 5 year old girls of age who are present at infante's belonging gardens to INTEGRA, JUNJI and Department of Education of the city of Viña del Mar. There evaluate skills of locomotion, manipulation and balance or stability by means of a guideline of observation made and validated in pre students of the Region del Maule, Chile, Vargas, C. (1). In the analysis of the information, across an analysis ANOVA with a level of significacncia alpha $=0,005$, in which there is demonstrated that statistically significant differences are not observed, in variable kind, in the total sample of the study, only some punctual cases to the interior of the educational estate JUNJI.
\end{abstract}

KEY WORDS: school Pre, fundamental bosses of movement, evaluation, significant differences, kind.

\title{
Dirigir correspondencia a:
}

Dr. Rodrigo Gamboa Jiménez.

Avda. El Bosque 1290, Santa Inés, Viña del Mar

CHILE

E-mail: rodrigo.gamboa@ucv.cl

\section{RECIBIDO 10-12-2010}

ACEPTADO 19-12-2010 